ORIGINAL ARTICLE

\title{
hTID-1 defines a novel regulator of c-Met Receptor signaling in renal cell carcinomas
}

\author{
E Copeland, S Balgobin ${ }^{1}$, CM Lee ${ }^{1}$ and M Rozakis-Adcock \\ Department of Laboratory Medicine and Pathobiology, Medical Sciences Building, University of Toronto, Toronto, Ontario, Canada
}

The c-Met receptor tyrosine kinase (MetR) is frequently overexpressed and constitutively phosphorylated in a number of human malignancies. Activation of the receptor by its ligand, hepatocyte growth factor (HGF), leads to increased cell proliferation, motility, survival and disruption of adherens junctions. In this study, we show that hTid-1, a DNAJ/Hsp40 chaperone, represents a novel modulator of the MetR signaling pathway. hTid-1 is a co-chaperone of the Hsp70 family of proteins, and has been shown to regulate a number of cellular signaling proteins including several involved in tumorigenic and apoptotic pathways. In this study we demonstrate that hTid-1 binds to unphosphorylated MetR and becomes dissociated from the receptor upon HGF stimulation. Overexpression of the short form of hTid-1 (hTid-1 $\mathrm{S}$ ) in 786-0 renal clear cell carcinomas (RCCs) enhances MetR kinase activity leading to an increase in HGF-mediated cell migration with no discernible effect on cell proliferation. By contrast, knockdown of hTid-1 markedly impairs both the onset and amplitude of MetR phosphorylation in response to HGF without altering receptor protein levels. hTid-1-depleted cells display defective migratory properties, coincident with inhibition of ERK/MAP kinase and STAT3 pathways. Taken together, our findings denote hTid-1 $\mathbf{S}_{S}$ as an essential regulatory component of MetR signaling. We propose that the binding of hTid-1 $\mathbf{s}_{\mathrm{S}}$ to MetR may stabilize the receptor in a ligand-competent state and this stabilizing function may influence conformational changes that take place during the catalytic cycle that promote kinase activation. Given the prevalence of HGF/MetR pathway activation in human cancers, targeted inhibition of hTid-1 may be a useful therapeutic in the management of MetR-dependent malignancies.

Oncogene (2011) 30, 2252-2263; doi:10.1038/onc.2010.601; published online 17 January 2011

Keywords: hTid-1; molecular chaperones; c-Met receptor tyrosine kinase; renal cell carcinoma; cell migration

Correspondence: Dr M Rozakis Adcock, Department of Laboratory Medicine and Pathobiology, Medical Sciences Building, University of Toronto, Faculty of Medicine, 1 King's College Circle, Room 6238A, Toronto Ontario, Canada M5S 1A8.

E-mail: maria.rozakis@utoronto.ca

${ }^{1}$ These authors contributed equally to this work.

Received 11 May 2010; revised 10 November 2010; accepted 30

November 2010; published online 17 January 2011

\section{Introduction}

hTid-1, a DnaJ chaperone protein of the Hsp40 family, is the human homologue of the drosophila tumor suppressor Tid56, the product of the lethal tumorous imaginal disc gene $(l(2) t i d)$. In drosophila, homozygous recessive mutations of $l(2)$ tid cause defects in differentiation and morphogenesis of larval imaginal discs leading to neoplastic growth of these cells into lethal tumors (Kurzik-Dumke et al., 1992, 1995). The human TID1 gene gives rise to two major alternative splice isoforms: a $43-\mathrm{kDa}$ long form $\mathrm{hTid}-\mathrm{l}_{\mathrm{L}}$ and a shorter $40 \mathrm{kDa}$ hTid-1 $\mathrm{S}$ variant, both of which are widely expressed (Trentin et al., 2001; Yin and Rozakis-Adcock, 2001). All hTid-1 isoforms consist of a $N$-terminal DNA 'J-domain', which is a highly conserved region present in all DNA J/Hsp40 family members that mediates binding to Hsp70 heat shock proteins (Hsps) (Wall et al., 1994) (Figure 2a). Following the DNA J-domain, is a glycine/phenylalanine rich $(\mathrm{G} / \mathrm{F})$ hinge region and a less conserved cysteine (Cys)-rich domain of $\sim 75$ aa in length, which is thought to facilitate the stabilization of target proteins with Hsp70/90 heterocomplexes (Banecki et al., 1996). The $\mathrm{COOH}$-terminal region of all DNA $\mathrm{J}$ proteins is the least conserved and is responsible for conferring substratebinding specificity.

hTid-1 has been implicated in the control of a number of signaling pathways including those involved with cell growth, survival, migration and tumor development. Several examples include the binding of hTid-1 to Ras p120 GTPase activating protein (GAP), a negative regulator of Ras (Trentin et al., 2001), binding and inhibition of NF- $\mathrm{BB}$ leading to growth arrest and cell death (Cheng et al., 2002, 2005), and suppression of Jak/STAT signaling though an interaction with Jak2 and interferon- $\gamma$ receptor (Sarkar et al., 2001). In addition, hTid-1 has been shown to associate with tumor suppressor proteins such as Patched and adenomatous polyposis coli (APC) proteins linked to the development of basal cell carcinoma and colorectal cancer, respectively (Kurzik-Dumke and Czaja, 2007; Wakabayashi et al., 2007). More recently, we identified the von Hippel-Lindau tumor suppressor (pVHL) as a novel binding partner of hTid-1 (Bae et al., 2005). Mutations in pVHL are found in a majority of sporadic renal cell carcinomas, hemangioblastomas and pheochromocytomas. The majority of these mutations hinder the ability of pVHL, a component of the E3 ubiquitin 
ligase complex ECV, to target hypoxia inducible factor $\alpha(\mathrm{HIF} \alpha)$ for polyubiquitinated degradation (Ohh et al. 1999). HIF $1 \alpha$ is a transcription factor that activates a number of genes involved in adaptation to hypoxia, including vascular endothelial growth factor (VEGF) and transforming growth factor $\alpha(T G F \alpha)$ (Iliopoulos et al., 1996). We have reported that recombinant hTid-1 through direct association with pVHL, enhances pVHL/HIF $1 \alpha$ complex formation and promotes HIF $1 \alpha$ degradation (Bae et al., 2005). Accordingly, hTid-1 is able to inhibit VEGF expression and angiogenesis both in vitro and in vivo.

hTid-1 is also known to interact with and influence the activity of receptor tyrosine kinases (RTKs), including ErbB-2, Trk and muscle-specific kinase MuSK (Kim et al., 2004; Liu et al., 2005; Linnoila et al., 2008). The interaction between ErbB-2 and Tid-1 was first described by Kim et al., who reported that exogenenous hTid-1 expression promotes the ubiquitination and degradation of ErbB-2 in human breast cancer cell lines and suppresses the in vitro and in vivo growth of ErbB2-dependent mammary carcinomas (Kim et al., 2004). The Trk family (TrkA, -B and -C) of RTKs have also been identified as hTid-1 binding proteins. Trk RTKs are activated by neurotrophins such as nerve growth factor and brain-derived neurotrophic factor. In this instance, hTid-1 inducibly associates with activated Trk receptors and promotes nerve growth factor-dependent neurite differentiation in nnr5-TrkA cells, coincident with sustained ERK/MAP kinase activity (Liu et al., 2005). More recently, hTid-1 was shown to complex with MuSK, a major component of the agrin receptor, and established as an essential intermediate in postsynaptic formation and transmission at neuromuscular junctions (Linnoila et al., 2008).

In this study we show that hTid-1 serves as an essential regulator of the c-Met receptor tyrosine kinase (MetR), a member of the RTK family that is activated by the hepatocyte growth factor/scatter factor (HGF/ $\mathrm{SF})$. Although a previous study by Schaaf et al. reported the binding of the oncogenic fusion protein, tpr-met, to the precursor form of hTid-1, the functional significance of this interaction was not addressed (Schaaf et al., 2005). MetR is overexpressed and hyperactivated in a number of human cancers including gliomas, osteosarcomas, breast and prostate carcinomas (Rong et al., 1994; Benvenuti and Comoglio, 2007) and often associated with tumor metastasis and poor clinical prognosis (Ferracini et al., 1995; Hov et al., 2004). It is noteworthy that both MetR and its ligand HGF have been shown to be overexpressed in renal clear cell carcinomas (RCCs), tumors often associated with hereditary or spontaneous loss of pVHL (Natali et al., 1996; Pennacchietti et al., 2003). Moreover, biochemical studies have linked inactivation of VHL in RCCs with constitutive tyrosine phosphorylation of MetR leading to disruption of adherens junctions and the acquisition of an invasive cell phenotype (Nakaigawa et al., 2006). Accordingly, MetR activity in RCCs can be inhibited by exogenous expression of wild-type VHL (Koochekpour et al., 1999). In light of the many levels at which the hTid-1 and VHL pathways coincide, we sought to investigate the contribution of hTid-1 in the regulation of the Met receptor-signaling pathway.

\section{Results}

\section{hTid-1 $1_{S}$ and $h$ Tid- $1_{L}$ modulate Met $R$ phosphorylation} levels independent of $p V H L$

We first set out to determine if hTid-1 influences pVHLmediated inhibition of MetR phosphorylation by overexpressing hTid-1 in both 786-0 RCC parental cells (786-0-VHL) and their stable transformants expressing exogenous wild-type VHL (786-0 + VHL) (Figure 1a). Adenoviruses expressing hTid-1 $\mathrm{S}($ Ad-hTid-1 $\mathrm{S})$, hTid-1 $\mathrm{L}_{\mathrm{L}}$ (Ad-hTid-1 L $_{\text {) }}$ or GFP alone (Ad-GFP) were generated and used to transduce RCC cells. Virally infected cells were serum-starved overnight and either left untreated or stimulated with $40 \mathrm{ng} / \mathrm{ml} \mathrm{HGF}$ for $10 \mathrm{~min}$. The use of this concentration of HGF results in robust autophosphorylation of MetR in 786-0 parental cells, as determined by immunoblotting for the activation loop phosphotyrosines 1234 and 1235 (pMet) (Figure 1a). Consistent with the data from Nakaigawa et al. (Nakaigawa et al., 2006), HGF-stimulated MetR phosphorylation was markedly inhibited in VHL-reconstituted 786-0 cells compared with parental 786-0 cells (Figure 1a, lane 2 vs lane 8). We found that overexpression of either Ad-hTid-1 $\mathrm{S}$ or Ad-hTid-1 had no discernible effect on pMet levels in the VHLproficient 786-0 cells. By contrast, overexpression of hTid- $1_{S}$ in 786-0 parental cells resulted in increased pMet levels in response to HGF treatment compared with GFP controls, whereas $\mathrm{hTid}-1_{\mathrm{L}}$ had a slight inhibitory effect. It should be pointed out that this change in phosphorylation status was not associated with changes in MetR protein levels.

To assess whether these findings represented a cellspecific idiosyncrasy, similar experiments were performed in the VHL-null invasive breast carcinoma cell line MDA-MB-231(Zia et al., 2007). We were able to confirm that $\mathrm{hTid}-1_{\mathrm{S}}$ expression enhanced, whereas hTid-1 ${ }_{\mathrm{L}}$ inhibited MetR phosphorylation in response to $\mathrm{HGF}$ (Figure 1b). By contrast, overexpression of either hTid-1 $_{S}$ or hTid-1 $_{\mathrm{L}}$ in A549 cells, a VHLproficient lung carcinoma cell line, had no impact on MetR activity (Figure 1c). Taken together, these findings suggest that both isoforms of hTid-1 have a modulatory effect on MetR activation in VHL null cells. In light of this observation, all further experiments were performed using 786-0 parental cells.

\section{hTid-1 proteins bind to unphosphorylated Met $R$}

To determine whether hTid-1 affects MetR activation through an interaction with the receptor, complex formation was first assessed using GST-Tid-1 fusion proteins. (Figure 2). Confluent layers of 786-0 RCC cells were serum-starved overnight and the following day the cells were either left untreated or stimulated with HGF for 10 minutes. Lysates from both untreated and HGF 
786-0

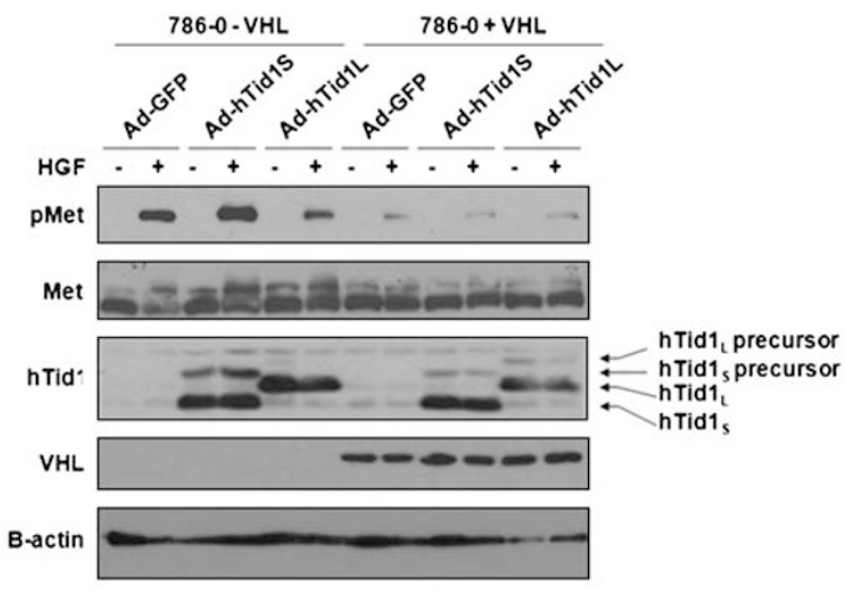

b

MDA-MB-231

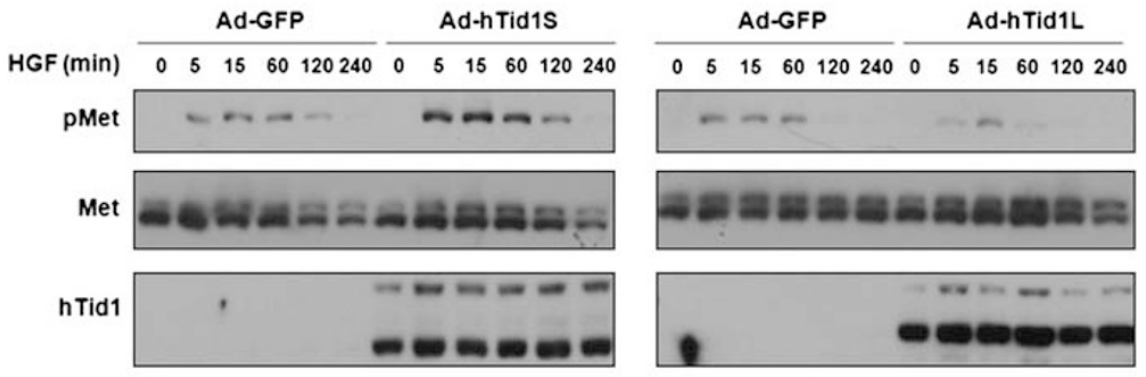

c

A549
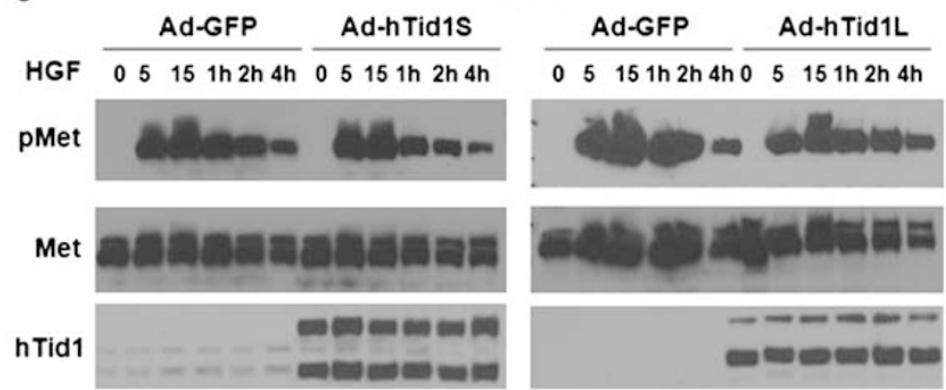

Figure 1 hTid-1 modulates MetR tyrosine phosphorylation. (a) 786-0 parental RCC cells (786-0-VHL) and 786-0 cells in which VHL has been reintroduced (786-0 + VHL) were infected with adenoviruses expressing either GFP alone (Ad-GFP), hTid- $1_{\mathrm{S}}\left(\right.$ Ad-hTid-1 $1_{\mathrm{S}}$ ) or hTid-1 $\mathrm{L}_{\mathrm{L}}\left(\right.$ Ad-hTid-1 $\left.\mathrm{L}_{\mathrm{L}}\right)$ as indicated. Cells were serum-starved overnight and either left untreated $(-)$ or treated with $40 \mathrm{ng} / \mathrm{ml} \mathrm{HGF}(+)$. Whole-cell lysates were immunoblotted with antibodies specific to phospho-Met (Tyr1234/1235 in the activation loop; pMet), total Met, hTid-1, VHL and $\beta$-actin. Met antibodies detect Met molecules of $170 \mathrm{kDa}$ (precursor) and $140 \mathrm{kDa}$ (mature $\beta$-chain). (b) MDA-MB-231 cells were infected and treated as in panel a, except that cells were treated with HGF over the indicated time course. (c) Experiment was repeated as in panel b using A549 lung cancer cells.

treated 786-0 RCC cells were incubated with either GST alone or the GST-full length hTid-1 s construct. The resulting complexes were subjected to western blot analysis using either anti-Met receptor or anti-phosphotyrosine 4G10 specific antibodies. To ensure cell lysates were incubated with equivalent amounts of GST proteins, the lower portion of the sodium dodecyl sulfate-PAGE gel was stained with Coomassie blue. (Figure 2b). An interaction between the recombinant GST-hTid-1 s $_{\text {S }}$ and MetR was observed in both serumstarved and HGF-treated lysates. Probing with 4G10 antiphosphotyrosine antibodies revealed that $\mathrm{HGF}$ mounts robust activation of the MetR (Figure 2b upper panel, lane 1 and 2), yet no discernible interaction between GST-hTid-1 and phosphorylated MetR was observed, indicating that hTid-1 preferentially binds to Met receptors in the kinase-inactive state.

The next step was to delineate which domain(s) of hTid-1 confer binding to MetR. To this end, three deletion constructs, each expressing a key hTid-1 domain, were expressed as GST fusion proteins in bacteria: (1) hTid1-J domain, the region responsible for mediating DNAJ/Hsp70 protein interactions, (2) $\mathrm{G} / \mathrm{F} /$ CYS-rich domain, a zinc-finger-like domain in the central region of hTid-1 and (3) hTid1-C-terminus, the region implicated in confering substrate binding 

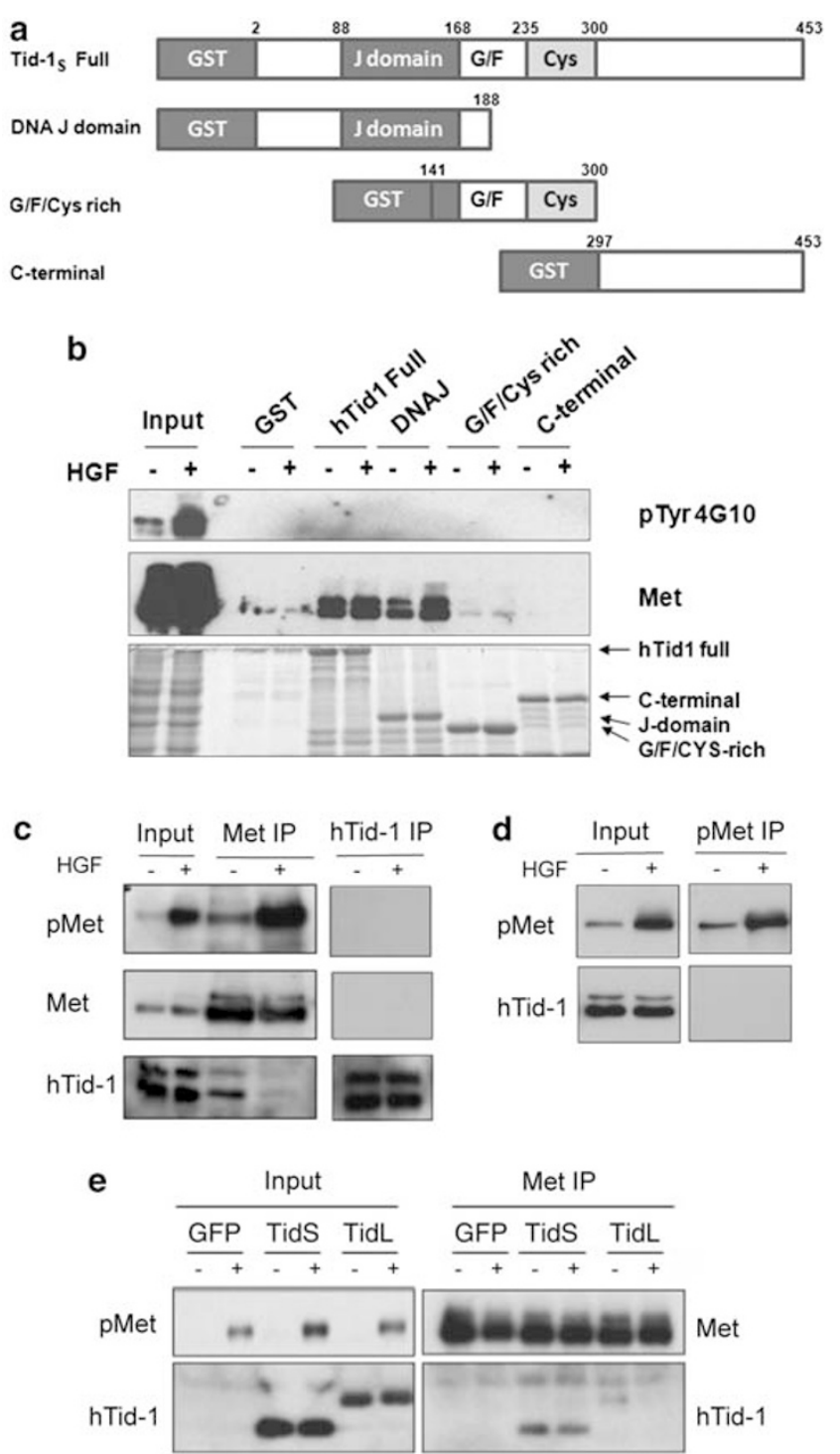

Figure 2 The $\mathbf{J}$ domain of hTid-1 selectively interacts with kinaseinactive MetR. (a) A schema depicting the GST constructs used in the GST pull-down experiments: full-length Tid-1 construct, a J-domain construct, a $\mathrm{G} / \mathrm{F} / \mathrm{Cys}$-rich and $C$-terminal construct. (b) 786-0 RCC cells were serum-starved and either left untreated $(-)$ or treated with $40 \mathrm{ng} / \mathrm{ml}$ HGF $(+)$. Lysates were incubated with the indicated GST proteins and the resulting complexes were subjected to immunoblotting with specific antibodies to MetR, and a phosphotyrosine specific antibody (pTyr 4G10). To ensure equal amounts of GST proteins used, the SDS-PAGE gel was stained with Coomassie blue (bottom panel). (c) 786-0 RCC cells were serum-starved and either left untreated $(-)$ or treated with HGF $(+)$. Cell lysates were immunoprecipitated with Met or hTid-1 antibodies and immune complexes were resolved by SDS-PAGE and analyzed by western blotting using antibodies specific to phospho-Met (Tyr1234/1235 in activation loop, pMet), total Met and hTid-1. (d) Cell lysates processed as shown in panel c were immunoprecipated with pMet antibodies and immune complexes probed with Tid-1 and pMet antibodies. (e) 786-0 Tid 1 null cells were infected with adenoviruses expressing either Ad-GFP, Ad-hTid-1 ${ }_{S}$ or Ad-hTid-1 $\mathrm{L}_{\mathrm{L}}$ and stimulated with HGF as indicated. Cell lysates were immunoprecipitated with Met antibodies and immunoblotted with either total Met, Tid-1 or pMet antibodies.
(Figure 2a). 786-0 cell lysates that had either been left untreated or stimulated with HGF were incubated with the indicated GST-hTid-1 fusion proteins and complexes were subsequently subjected to western blot analysis. Interestingly, immunoblotting with anti-MetR antibodies revealed that the interaction between hTid-1 and MetR is mediated through the J-domain of Tid1 and not the $C$-terminal substrate-binding domain (Figure 2b). The light MetR immunoreactive band present in the GST- Tid1-G/F/Cys-pull-downs likely reflect a nonspecific interaction with the GST moiety, as similar nonspecific signals appear in GST controls.

To test whether MetR and hTid-1 associate in vivo, co-immunoprecipitation experiments were performed in 786-0 cells (Figure 2c). Cell lysates from both serum-starved and HGF-stimulated cells were immunoprecipitated with anti-MetR antibodies and the resulting immunocomplexes were probed with anti-hTid-1 antibodies. Our results revealed that although both isoforms of hTid-1 were present in MetR immune complexes, the short isoform of hTid-1 was preferentially associated with MetR in vivo. Similar observations were made with exogenously expressed Ad-hTid-1 ${ }_{S}$ and

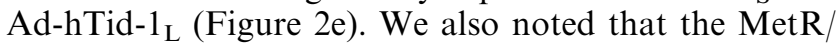
hTid-1 complex preformed under basal conditions was dissociated in response to HGF (Figure 2c, lane 3 vs lane 4). Interestingly, overexpression of recombinant Ad-hTid-1 $1_{S}$ but not Ad-hTid-1 L $_{\text {displayed prolonged }}$ association with MetR, which may serve to promote signal amplification (Figure 2e). Our data suggest that both hTid-1 isoforms preferentially interact with the kinase inactive conformer of MetR and that ligand binding stimulates receptor conformational changes, which release MetR from the hTid-1 chaperone machinery. In support of this observation, immunoprecipitation assays using phosphospecific MetR antibodies that recognize the twin phosphotyrosines Tyr-1234/1235 in the activation loop of MetR clearly established that the activated receptor does not bind hTid-1 (Figure 2d).

It should be pointed out that immunoprecipitation assays with hTid-1 antibodies failed to demonstrate detectable binding between hTid-1 and MetR (Figure 2c, right panel). One possible explanation is that our polyclonal antibodies raised against hTid-1 disrupt hTid-1/MetR complexes, which is an observation we have previously reported for other Tid-1/ substrate interactions (Trentin et al., 2001).

The ability of MetR to serve as a direct binding partner of hTid-1 prompted us to examine the subcellular location at which this interaction occurs. Previous data from our laboratory and others have shown that hTid-1 is distributed not only within the mitochondrial compartment but found at the plasma membrane, cytosol and nucleus depending on the particular cell type (Sarkar et al., 2001; Trentin et al., 2001; Linnoila et al., 2008). Analysis of immunofluorescent staining patterns under serum-starved conditions revealed that hTid-1 and MetR exhibited a punctate distribution throughout the cytosolic compartment with areas of colocalization (Figure 3, top panels) that may represent the chaperoning of MetR molecules by 

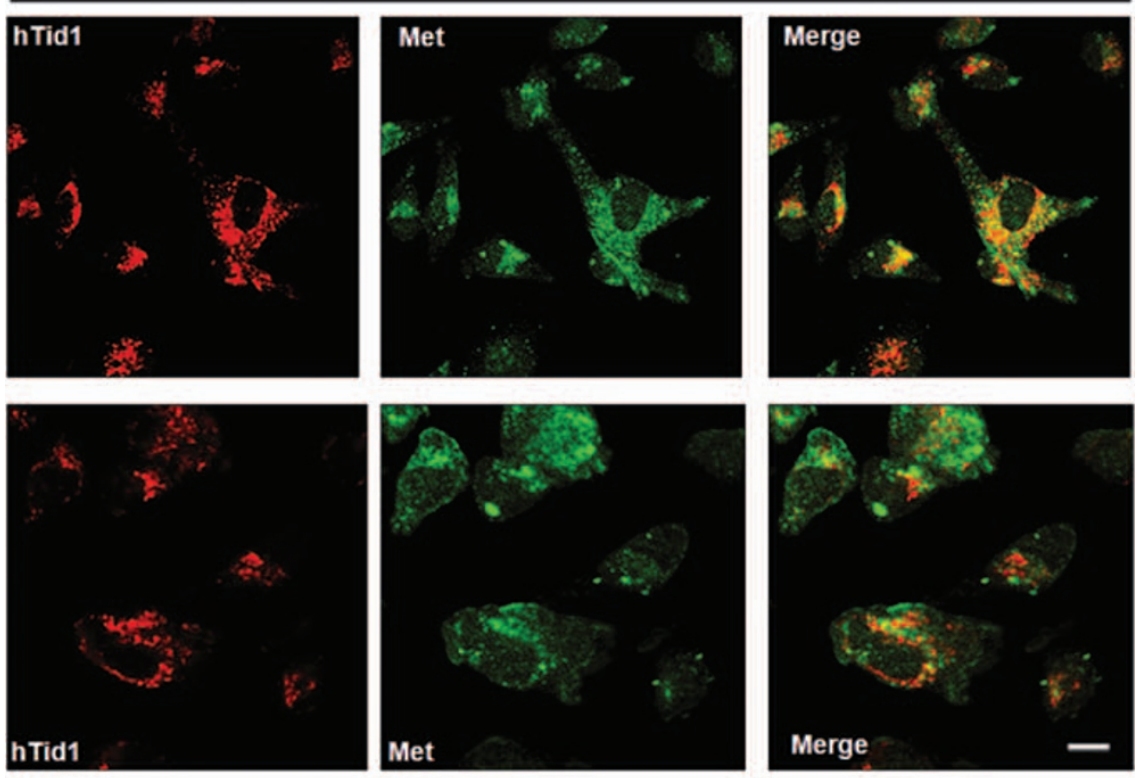

+ HGF

Scale bar $=10 \mu \mathrm{m}$

Figure 3 Localization of MetR with hTid-1. 786-0 cells were seeded on coverslips and either left untreated (-HGF) or stimulated with HGF ( + HGF) at $37^{\circ} \mathrm{C}$ for $10 \mathrm{~min}$. Coverslips were fixed in $2 \%$ PFA and stained with MetR and Tid-1 antibodies and images captured with a Zeiss LSM 410 Laser Scanning Confocal Microscope. Yellow staining represents colocalization between endogenous hTid-1 (red) and MetR (green).

hTid-1 within the biosynthetic pathway. On binding HGF, we observed that MetR accumulated in a perinuclear region of the cell (Figure 3, lower panels) and this shift in topology is consistent with previous studies demonstrating that MetR transits to perinuclear foci suggestive of association with late endosomes/ lysosomes (Hammond et al., 2001; Sangwan et al., 2008).

\section{hTid-1 ${ }_{S}$ enhances $H G F-$ mediated cell migration}

HGF is a potent motogen, mediating epithelial cell scattering, branching tubule formation and invasion of cells through a matrix (Birchmeier et al., 2003). Seeing as $\mathrm{hTid}-1_{\mathrm{S}}$ overexpression results in enhanced activation of MetR by HGF (Figure 1), we wanted to determine whether this would influence HGF-mediated migration. The first experimental approach used to examine migration was a transwell assay. 786-0 RCC cells were infected with Ad-hTid-1 $\mathrm{S}$, Ad-hTid-1 ${ }_{\mathrm{L}}$ or Ad-GFP viruses as control and following serum-starvation, 75000 cells were resuspended in DMEM with $0.5 \%$ bovine serum albumin (BSA) and plated in the upper chamber of a transwell filter. Using $\mathrm{HGF}$ as a chemoattractant in the lower chamber, the number of cells that migrated through the $8-\mu \mathrm{m}$ porous membrane was counted. After $24 \mathrm{~h}$ of incubation, the number of hTid- $1_{S}$ overexpressing cells that had migrated through the filter was significantly higher $(P<0.05)$ than the number of GFP-infected control cells (Figure 4). There was no significant difference between hTid-1 ${ }_{\mathrm{L}}$ overexpressing cells and control cells. This demonstrates that
hTid- $1_{\mathrm{S}}$ is able to promote $\mathrm{HGF}$-mediated migration and is consistent with the enhanced MetR activation that is observed with hTid-1 $1_{\text {S }}$ overexpression.

A wound closure assay was used as an alternative measure of cellular migration. 786-0 RCC cells were infected with Ad-GFP, Ad-hTid-1 $\mathrm{S}$ or Ad-hTid-1 viruses, grown to confluency and serum-starved overnight. The following day a uniform wound was made in the confluent layer of cells and the width of the wound following its formation was measured at the indicated time points. The cells were either left untreated or stimulated with HGF over the time course indicated. The wound in 786-0 cells infected with Ad-hTid-1s closed significantly faster than that of the control cells (Figure 5, $P<0.05$ ), which is consistent with the increased migration observed in the transwell assay. This effect was observed in the presence of serum-free media, as well as in HGF-treated 786-0 cells (Figures 5a and b) suggesting that hTid-1 ${ }_{S}$ may also be influencing additional motogenic signaling pathways other than $\mathrm{HGF} / \mathrm{MetR}$. Consistent with the results from the transwell migration assay there was no significant effect of $\mathrm{hTid}-1_{\mathrm{L}}$ on wound closure compared with control cells.

To determine whether hTid-1 has any effect on the growth rate of 786- 0 cells, cell proliferation assays were performed both in untreated (Figure 6a) and HGFstimulated (Figure 6b) cells. 786-0 cells were infected with adenoviruses expressing hTid-1 ${ }_{S}$, hTid- $1_{L}$ or a GFP control, serum-starved overnight and the following day (day 0) were counted and plated in 24-well plates. On each indicated day (up to 7 days post-infection) 

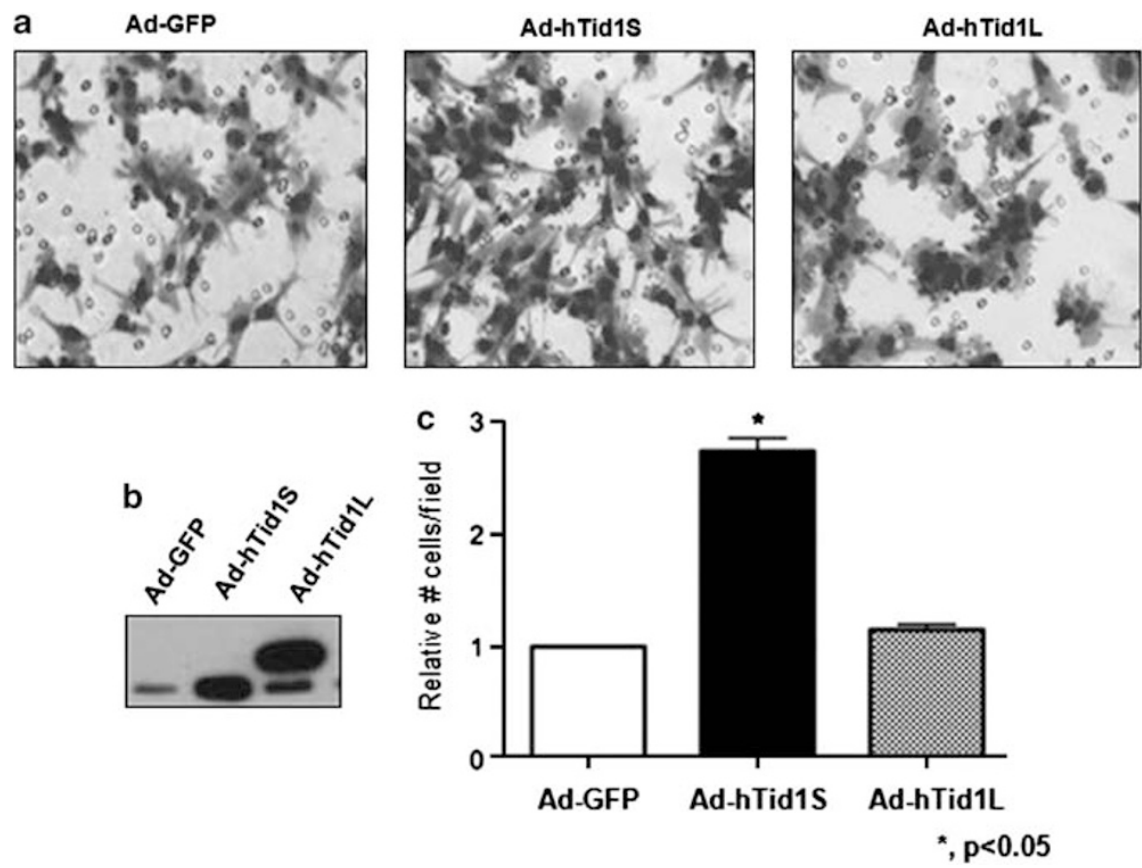

Figure 4 hTid- $1_{S}$ enhances HGF-mediated migration. (a) hTid-1 $1_{S}\left(\right.$ Ad-hTid- $1_{S}$ ) and $h$ hTid- $1_{L}$ (Ad-hTid- $1_{L}$ ) were overexpressed in 786-0 RCC cells via adenoviral infection. An adenovirus expressing GFP alone was used as a control (Ad-GFP). The cells were serumstarved overnight and plated in the upper chamber of a transwell chamber, using HGF as a chemoattractant in the lower chamber. After $24 \mathrm{~h}$ of incubation, the cells that had migrated were fixed and stained with Diff-Quik. Representative pictures from each filter are shown. (b) Lysates from cells infected simultaneously as those used for migration were immunoblotted with anti-hTid-1 to confirm efficient hTid-1 overexpression. (c) Migrated cells were counted in five individual fields and averaged. The results are pooled from three independent experiments. An unpaired $t$-test was used to determine significance between GFP and hTid-1 $\mathrm{S}_{\mathrm{S}}$ or hTid-1 $1_{\mathrm{L}}$ overexpressing cells. *, a $P$-value of $<0.05$ was considered significant.

triplicate wells for each adenoviral condition were trypsinized and the number of cells counted. Our data indicate that there was no significant difference in the proliferation rates of either $\mathrm{hTid}-\mathrm{1}_{\mathrm{S}}$ or $\mathrm{hTid}-1_{\mathrm{L}}$-infected cells compared with GFP controls, suggesting that hTid-1 has no effect on cellular proliferation in the 786-0 RCC tumor cell model.

hTid-1 modulates HGF-mediated MAPK phosphorylation Activation of both PI3K-mediated and MAPKmediated signaling pathways have been implicated in HGF-induced migration. To determine which of these pathways, if any, were responsible for mediating the increased migration in $\mathrm{hTid}-1_{\mathrm{S}}$ overexpressing cells, we examined the phosphorylation status of both Akt and Erk1/2. hTid-1 $1_{S}$ overexpression resulted in a robust HGF-dependent activation of Erk1/2 compared with control cells, whereas Akt phosphorylation at Ser473 was unchanged (Figure 7). These results demonstrate that $\mathrm{hTid}-1_{\mathrm{S}}$ enhances HGF-dependent MAPK activation, but not PI3K signaling, to promote cellular migration in 786-0 RCC cells.

\section{hTid-1 knockdown inhibits MetR activation and migration}

To add validity to the results obtained so far using hTid1 overexpression, we next sought to investigate whether hTid-1 proteins have a physiological role in c-Metdependent signaling by using RCC cells where hTid-1 expression was silenced. To this end, 786-0 cells stably expressing either a control nonspecific short interfering (si) RNA or a gene specific siTid-1 construct were serum-starved overnight and exposed to HGF ligand for varying time points. Whole-cell lysates were collected and western blotting was used to analyze phospho-Met (Tyr1234/Tyr1235, pMet), total MetR and hTid-1 levels (Figures $8 \mathrm{a}$ and $\mathrm{b}$ ). We found that in the absence of endogenous hTid-1 proteins there was a marked inhibition of MetR phosphorylation in HGF-treated cells, consistent with the idea that hTid-1 chaperone function was necessary for efficient ligand-dependent activation of MetR. We subsequently assessed whether hTid-1 silencing influences the migratory properties of 786-0 cells in response to HGF. Transwell migration assays performed using HGF as a chemoattractant revealed that stable depletion of endogenous hTid-1 proteins severly compromises $786-0$ migration $(\sim 70 \%$ inhibition) when compared with siRNA control cells (Figure 8b). Moreover, we observed that this suppression in cell motility correlated with an inhibition in Erk1/2 and STAT3 activation in response to HGF treatment (Figure 7c).

\section{Discussion}

Molecular chaperones, best known for their role in the biogenesis and folding of nascent polypeptides, have 

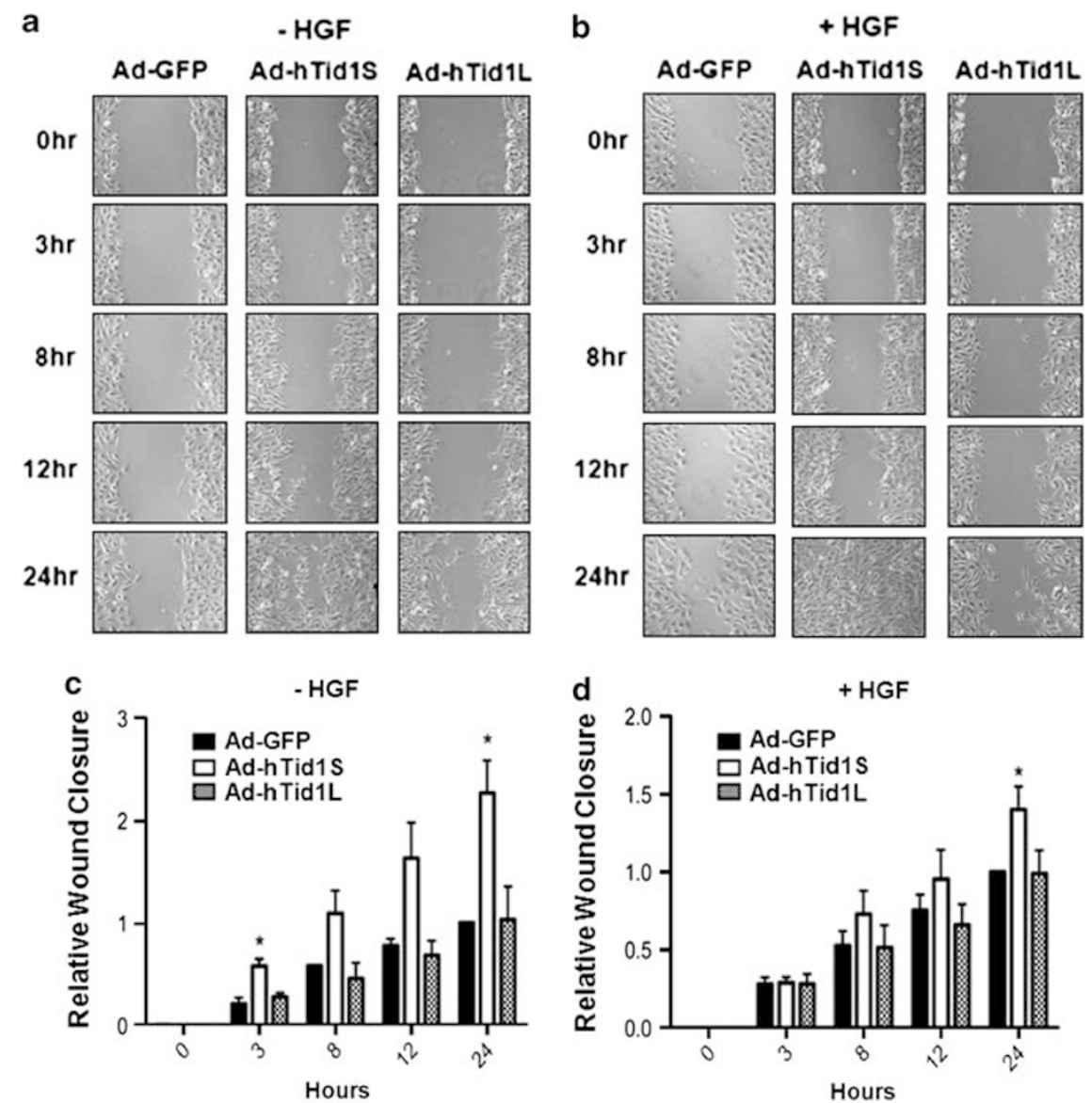

Figure 5 hTid- $1_{S}$ enhances HGF-mediated wound closure. (a) 786-0 RCC cells were infected with adenoviruses expressing either hTid-1 S $_{\mathrm{S}}$ (Ad-hTid-1 $_{\mathrm{S}}$ or hTid-1 $\mathrm{L}$ (Ad-hTid-1 $\mathrm{L}_{\mathrm{L}}$. A GFP expressing adenovirus (Ad-GFP) was used as a control. At 24-h post-infection, the cells were serum-starved for an additional $24 \mathrm{~h}$. A uniform wound was made in the confluent cell layer at $0 \mathrm{~h}$. The cells were then incubated with fresh serum-free media. (b) The same experiment was performed as shown in panel a, except that $5 \mathrm{ng} / \mathrm{ml} \mathrm{HGF}$ was added to the serum-free media at $0 \mathrm{~h}$. Pictures of the wound at specific locations were taken at the indicated time points. (c, d) The wound width was calculated using Simple PCI software, and the relative wound closure was calculated setting wound closure of the control condition at $24 \mathrm{~h}$ as 1 . The data presented is a representative experiment from three independent experiments. An unpaired $t$-test was used to calculate significance of either hTid-1 $\mathrm{S}_{\mathrm{S}}$ or hTid-1 $1_{\mathrm{L}}$ overexpressing cells compared with the control at each time point. *, a $P$-value of $<0.05$ was considered significant.

an integral role in numerous intracellular signaling pathways, including those involved with cell survival and growth regulation. Many of these chaperones were originally identified as Hsps and belong to one of four major families: Hsp 60, Hsp 70, Hsp 90 and Hsp100, which rely on accessory proteins or cochaperones (for example, DNA J/Hp40, Cdc37, Immunophilin) to determine their functional specificity (Frydman et al., 1994; Duina et al., 1996; Freeman et al., 1996; Hartl, 1996; Chang et al., 1997). Hsps have a critical role in protecting cells from environmental and physiologic stresses or pathological conditions that lead to the appearance of misfolded, mutated or otherwise damaged molecules (Jolly and Morimoto, 2000). As such, Hsp function in cancer cells is often co-opted to support malignant transformation, by serving as a biochemical buffer that permits aberrant functioning of mutated oncoproteins and escape from genotoxic cell death. Hsp70 and Hsp90 are known to govern the biological activity of a number of cancer-associated signaling proteins. Most of the known clients of Hsp90 can be divided into two categories, signaling kinases (for example, ErbB-2, IGF-1R, v-Src, PKR, Akt, Raf-1) and transcription factors (steroid hormone receptors, p53, HIF $1 \alpha$ ) (Pratt and Toft, 2003). Recruitment of Hsp90 to these substrates stabilizes their conformations, facilitating ligand binding, substrate phosphorylation or subsequent protein interactions. Contrary to the pro-tumorigenic and antiapoptotic properties of $\mathrm{Hsp} 70$ and Hsp90 proteins, hTid-1 encodes the first member of the DNAJ/Hsp40 chaperone family to be classified as a tumor suppressor (Trentin et al., 2001; Yin and Rozakis-Adcock, 2001; Chen et al., 2009; Ahn et al., 2010). Biochemical evidence suggests that hTid-1 may exert its antitumor function by interacting with and inhibiting the activity of mitogenic or oncogenic proteins such as human papillomavirus E7 oncoprotein (Schilling et al., 1998), Jak2 kinase (Sarkar et al., 2001) and ErbB-2 (Kim et al., 2004). Subsequent studies demonstrated that hTid-1 represses NFkB activity induced by HTLV-1 Tax and cytotoxic stimuli including $\mathrm{TNF} \alpha$, cisplatin and $\mathrm{MMC}$, by directly binding and 


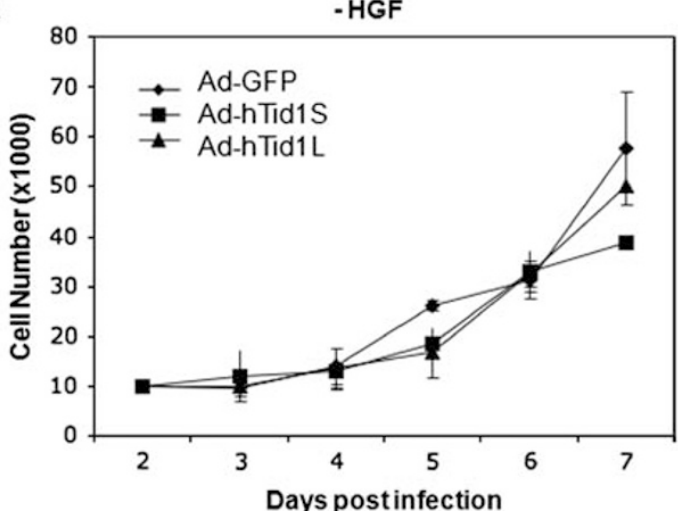

b

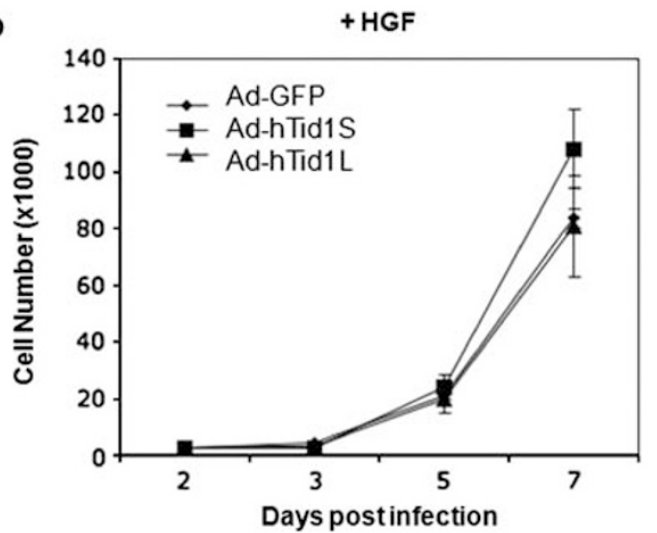

C

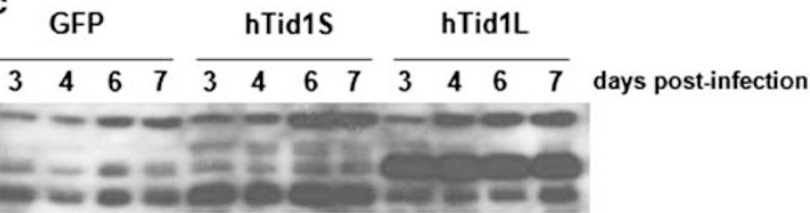

Figure 6 hTid-1 overexpression does not affect 786-0 cellular proliferation. (a) 786-0 cells were infected with adenoviruses expressing either GFP, hTid- $1_{\mathrm{S}}$ or $\mathrm{hTid}-\mathrm{l}_{\mathrm{L}}$, and serum-starved overnight. A total of 10000 cells/well were plated in 24-well plates on day 0 . Triplicate wells were counted with a haemocytometer on the indicated days post-infection. The mean and s.d. of each triplicate time point was determined. (b) Experiment was repeated as shown in panel a except 2500 cells/well were plated in each well of a 24-well plate and cells were treated with $100 \mathrm{ng} / \mathrm{ml} \mathrm{HGF}$. (c) Lysates from adenoviral infected 786-0 cells from panels a and $\mathbf{b}$ were subjected to immunoblot analysis using anti-hTid-1 antibodies.

inhibiting IKK kinase activity and in so doing modulating apoptotic signal transduction in mammalian cells. hTid-1 also has a direct bearing on the function of a number of tumor suppressor proteins such as VHL, p53 and adenomatous polyposis coli, however, in this instance it serves to promote their role in cell-death pathways by influencing several facets of their signaling capacity such as their subcellular localization, assembly into multiprotein complexes and/or coupling to the proteosomal machinery (Bae et al., 2005; Kurzik-Dumke and Czaja, 2007; Ahn et al., 2010; Qian et al., 2010).

The data presented herein introduces a novel role for hTid-1 in the regulation of c-Met receptor signaling in

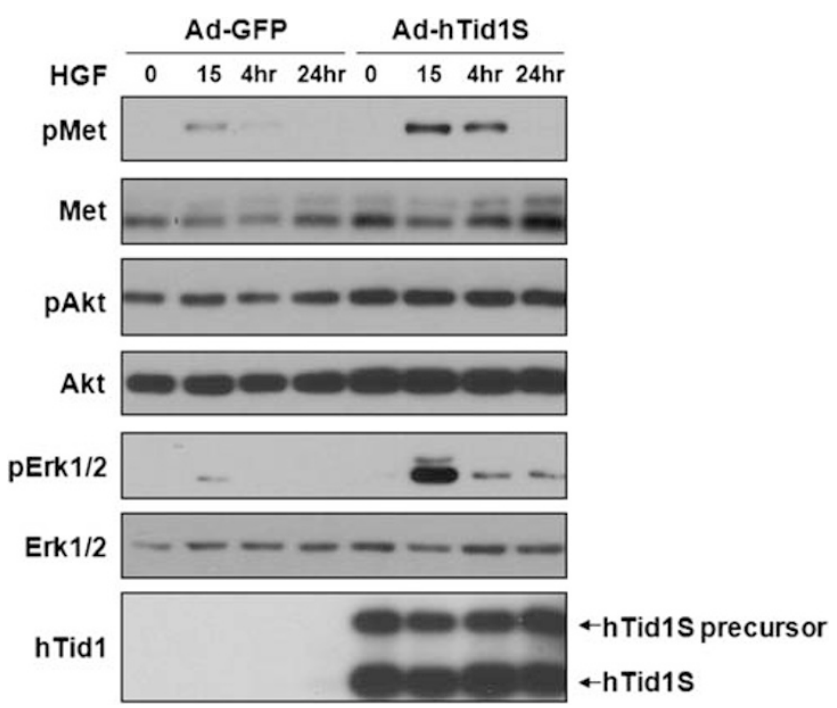

Figure 7 Enhanced motility is associated with increased Erk1/2 activation. 786-0 RCC cells were infected with adenoviruses expressing $\mathrm{hTid}-1_{\mathrm{S}}\left(\right.$ Ad-hTid- $1_{\mathrm{S}}$ ) or GFP as a control (Ad-GFP). The cells were serum-starved overnight and treated with HGF for the indicated time periods. Whole-cell lysates were resolved by SDS-PAGE and analyzed by western blotting with antibodies specific to phospho-Met (Tyr1234/1235; pMet), total Met, phospho-Erk1/2, total Erk1/2, pAkt, total Akt and hTid-1.

renal cell carcinomas which are often associated with hereditary or spontaneous loss of VHL. Biochemical studies indicate that VHL-negative RCCs display a heightened response to HGF-driven activities and correlate with increased cell motility, matrix invasion and tubulogenesis (Koochekpour et al., 1999; Peruzzi and Bottaro, 2006; Peruzzi et al., 2006; Zia et al., 2007). The source of this enhanced MetR signaling in VHLdefective RCCs is somewhat controversial with some groups pointing to changes in the activity of dowstream effectors such as HIF $2 \alpha, \beta$-catenin, E-cadherin and TIMPs, which synergize with HGF, while others observe changes in the activity of the c-Met receptor itself (Birchmeier et al., 2003). In this study, we provide evidence that hTid-1 influences the dynamics of c-Met receptor activation in VHL-null RCCs. hTid-1 seems to achieve this effect through a direct interaction with MetR in its catalytically inactive unbound state. Although MetR is found to co-immunoprecipitate with both long and short isoforms of hTid-1 under basal conditions, there is preferential binding to Tid- $1_{\mathrm{S}}$. Similar observations were noted for the MuSK receptor that selectively interacts with hTid-1 (Linnoila et al., 2008). Significantly, we and others have reported differential activities of these two Tidl isoforms in cancer cell lines (Syken et al., 1999, 2003; Edwards and Munger, 2004; Trentin et al., 2004). In the context of RCC cells, overexpression of $\mathrm{hTid}-1_{\mathrm{S}}$ in parental 786-0 cells is seen to enhance HGF-mediated MetR autophosphorylation on tyrosine residues Y1234 and Y1235 within the activation loop of the TK domain without altering steady levels of the protein, whereas hTid- $1_{\mathrm{L}}$ shows a modest inhibition of MetR activity. Moreover, siRNA-mediated depletion of endogenous hTid-1 

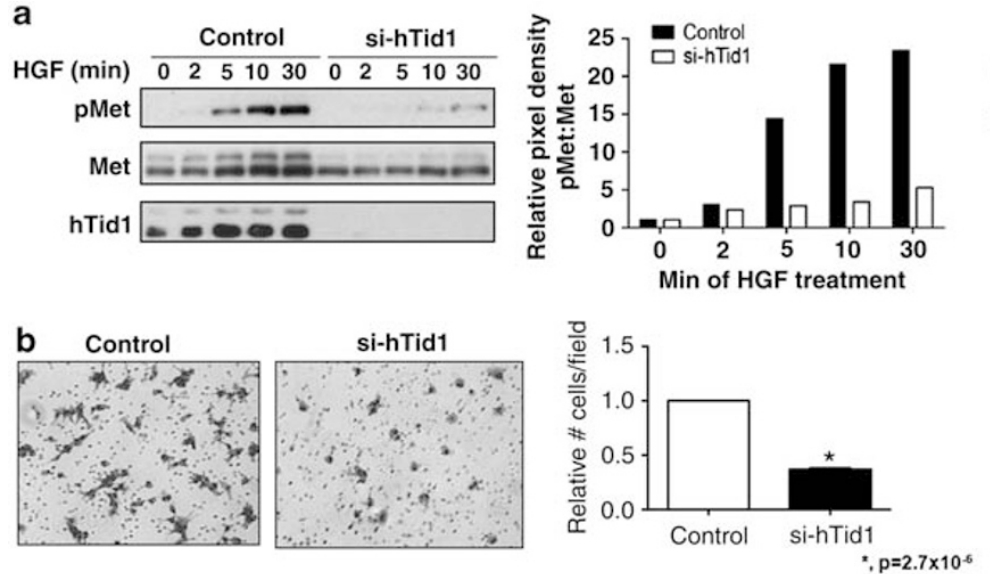

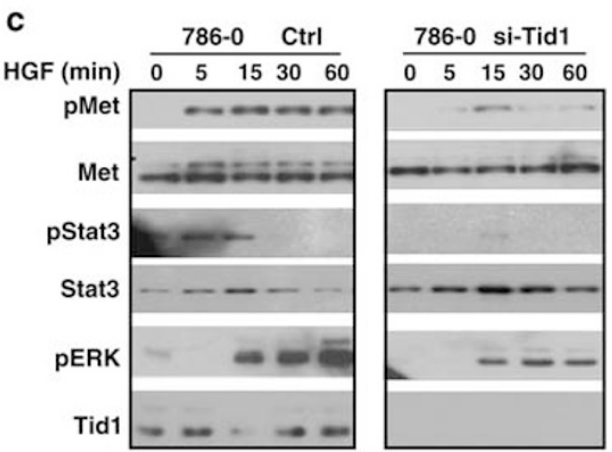

Figure 8 hTid-1 knockdown inhibits Met activation and HGF-mediated migration. (a) Left panel, 786-0 RCC cells that had been stably infected with either a control lentivirus or a si-hTid-1 expressing lentivirus were serum-starved overnight and treated with HGF for the indicated time points. Whole-cell lysates were collected and resolved by SDS-PAGE. Western blotting was used to analyze phospho-Met (Tyr1234/Tyr1235, pMet), total Met and hTid-1 levels. Right panel, densitometric analysis was used to determine the degree of Met phosphorylation relative to total Met levels and is shown as the relative pixel density of pMet:Met compared with untreated cells. (b) Left panel, 786-0 cells that have been stably infected with either a control lentivirus or a si-hTid-1 expressing lentivirus were serum-starved overnight and used in a transwell migration assay. A total of $7.5 \times 10^{5}$ cells were plated in the upper chamber with HGF in the lower chamber. Cells were allowed to migrate for $24 \mathrm{~h}$, and representative pictures for each filter is shown. Right panel, The number of cells that migrated was counted in five individual fields/filter and the average number of cells/field was calculated, and is shown as a ratio to the number of cells that migrated in the control condition. The data shown represent pooled data from three independent experiments. An unpaired $t$-test was used to determine significance. $*$, a $P$-value of $<0.05$ was considered significant. (c) hTid-1 knockdown inhibits signaling through Erk1/2 and STAT3. 786-0 RCC cells that had been stably infected with either a control lentivirus or a si-hTid-1 expressing lentivirus were serum-starved overnight and treated with $10 \mathrm{ng} / \mathrm{ml} \mathrm{HGF}$ for the indicated time points. Whole-cell lysates were prepared and subjected to immunoblot analysis using antibodies specific for phosphoMet (Tyr1234/1235, pMet), total Met, pSTAT3, total STAT3, phospho-Erk1/2 (pErk1/2), hTid-1 and $\beta$-actin.

proteins strongly compromises both the onset and amplitude of HGF-stimulated phosphorylation of the receptor, establishing that the protein chaperoning function of hTid-1 $_{\mathrm{S}}$ is essential for ligand-induced conformational transitions in the kinase domain that lead to receptor activation. However, it should be pointed out that the regulatory impact of hTid-1 on MetR was not observed in VHL proficient $786-0$ or A549 lung carcinoma cell lines. Thus, it is possible that hTid-1 functions through an alternative mechanism to that proposed for the regulation of MetR activation by VHL, which interestingly is observed only under high cell density conditions. Another possibility is that in VHL-proficient cells, MetR requires only limited assistance from Tid- $1_{S}$ to maintain the kinase domain in a readily activatable state; whereas under VHL-null conditions the receptor may adopt a more unstable or labile conformation that leads to a higher requirement for Tid-1 function in the stabilization and/or allosteric activation of MetR.

Our GST-pull-down assays revealed that binding of hTid-1 to MetR is mediated through its J-domain, which was somewhat suprising given that hTid-1 has been reported to interact with other RTK client proteins such as ErbB2, Trk and MuSK via its $C$-terminal substrate-binding region (Kim et al., 2004; Liu et al., 2005; Linnoila et al., 2008). In these instances, the authors propose that the effect of hTid-1 on RTK function is dependent on its ability to couple to Hsp70 chaperones via its DNAJ domain. Coincidently, MetR is not unique on its reliance on the hTid-1 DNAJ region for complex formation, as $\mathrm{I} \kappa \mathrm{B}$ and $\mathrm{IKK}$ interaction domains on hTid-1 have also been mapped to the J domain (Cheng et al., 2005). Currently there is no published evidence suggesting that MetR is a Hsp70 substrate, and our own attempts at detecting an interaction between the two proteins have been unsuccessful (data not shown). However, there are a number of independent studies that provide support for MetR as a Hsp90 client protein. Inhibition of Hsp90 function via geldanamycin treatment has been shown to downregulate MetR protein expression levels suggesting that Hsp90 is necessary for stabilization of MetR in its mature conformation (Webb et al., 2000; Maulik et al., 2002; Lesko et al., 2007). Disruption of Hsp90 function has also been associated with inhibition of MetR activation by HGF (Koga et al., 2007). It is therefore possible that hTid-1 modulates MetR phosphorylation as a part of a larger Hsp90 multicomponent chaperone complex. At present, however, we cannot rule out the possibility that hTid-1 can chaperone client proteins such as MetR in its own right, as well as indirectly through modulation of HSP70/Hsp90 function.

In this study, we also addressed the role of hTid-1 on downstream MetR signaling in RCC cells. The increased MetR phosphorylation observed upon hTid-1 $\mathrm{S}$ overexpression translated into enhanced HGF-mediated cell migration. This correlated with increased activation of the MAPK signaling cascade as measured by Erk1/2 phosphorylation. The activities of both PI3K and 
MAPK signaling pathways are important for mediating HGF-driven cell motility. However, in this context, we saw no apparent difference in PI3K-dependent AKT phosphorylation at Ser473 when hTid-1 s was overexpressed, despite the fact that an overall enhancement in MetR activation was observed. Our results are in alignment with independent reports demonstating that hTid-1 $1_{\mathrm{S}}$ overexpression selectively modulates the MAPK branch of ErbB2 and Trk receptor signaling pathways with no discernible effect on the activity of AKT (Kim et al., 2004; Liu et al., 2005). Our findings also support the idea that there are additional signaling networks that impact cell migration that are likely under the influence of hTid-1. Notably, depletion of endogenous hTid-1 proteins leads to a profound inhibition of cell motility in HGF-treated cells, which correlates with STAT3 inactivation. Furthermore, we also note that hTid-1 S $_{\mathrm{S}}$ overexpression provokes a marked enhancement in the migratory potential of 786-0 cells even under serum-starved conditions, indicating that hTid-1 proteins can function through HGF-independent pathways to influence cell migration, however, the precise mechanisms remain to be elucidated.

In summary, our results point to $\mathrm{hTid}^{-1_{\mathrm{S}}}$ as an essential allosteric activator of the c-Met receptor in RCCs. Evidence is accumulating that hTid-1 proteins have disctinct chaperoning functions that impact a large number of signaling effectors implicated in cellfate determination, cellular differentiation, proliferation, survival and invasion/metastasis. The mechanism of action by which hTid-1 regulates the activity of these client proteins will be key areas for future research.

\section{Materials and methods}

\section{Cell lines and reagents}

The human RCC cell line 786-0 has a germ-line VHL frameshift mutation and does not express VHL. Parental 786-0 and 786-0 recombinants, in which VHL has been stably reintroduced (786-O + VHL) were generously provided by $\mathrm{Dr} \mathrm{M}$ Ohh (University of Toronto). The NSCLC cell line A549 was kindly provided by Dr M Tsao (University of Toronto). HEK293T and MDA-MB-231 cells were purchased from ATCC (Manassas, VA, USA). Antibodies to phospho-Met Y1234/5, phospho-Akt Ser 473, Akt, phospho-STAT3, STAT3, phosphotyrosine residues (4G10) and HRP-linked secondary antibodies were purchased from Cell Signaling Technologies (Beverly, MA, USA). Met (C28; C12) antibodies were from Santa Cruz Biotechnology (Santa Cruz, CA, USA); Phospho-Erk1/2 and Erk1/2 antibodies from Zymed Laboratories (San Francisco, CA, USA); VHL antibodies from BD Biosciences (San Jose, CA, USA). $\beta$-actin antibody and human recombinant HGF were purchased from Sigma (Oakville, ON, Canada). The rabbit polyclonal antibodies to hTid-1 were generated as described (Trentin et al., 2001).

\section{Adenoviral infections}

Adenoviruses generated by cloning the complementary DNAs of full-length hTid-1 $\mathrm{S}$ and hTid-1 $\mathrm{L}_{\mathrm{L}}$ downstream of the CMV promoter in pAdTrack-CMV have been previously described (Trentin et al., 2001).

\section{Lentiviral production and infections}

The hTid-1 interfering RNA (RNAi) construct in the lentivirus vector pLL3.7 along with the parental pLL3.7 vector was kindly provided by $\mathrm{Dr}$ Isaiah $\mathbf{J}$ Fidler (The University of Texas). The pLL3.7/hTid-1 RNAi plasmid was contransfected into the $293 \mathrm{~T}$ packaging cell line with a 3rd generation packaging system consisting of the vectors pMDLg/pRRE (gag/pol elements), pRSV-REV and pMD.G (envelope elements). The viral supernatant was harvested 48 -h post transfection and was used to transduce the 786-0 RCC cell line in the presence of $8 \mu \mathrm{g} / \mathrm{ml}$ polybrene.

\section{GST fusion-protein preparation and pull-down experiments}

GST-fusion constructs and pull-down assays have been previously described (Trentin et al., 2001). Proteins bound to GST-fusion proteins were subjected to western blot analysis with the indicated antibodies.

\section{Immunoprecipitation}

For immunoprecipitation experiments cells were lysed in modified RIPA, and the supernatant was pre-cleared with $100 \mu \mathrm{l}$ of a $10 \%$ protein-A agarose slurry. Protein determination was done via a Bradford assay on the pre-cleared lysate. A measure of $800 \mu \mathrm{g}$ of cell lysate was immunoprecipitated with $2 \mu \mathrm{g}$ of the indicated antibody and $100 \mu \mathrm{l}$ of a $10 \%$ protein-A agarose slurry overnight at $4{ }^{\circ} \mathrm{C}$. The protein-A agarose beads were washed $3 \times$ with PLC buffer before loading onto an sodium dodecyl sulfate-PAGE gel.

\section{Immunofluorescence}

786-0 RCC were plated on BD 8-well chamber slides at 20000 cells per well and starved in serum-free DMEM for $48 \mathrm{~h}$. For HGF stimulation, cells were incubated with $40 \mathrm{ng} / \mathrm{ml}$ of $\mathrm{HGF}$ (Peprotech, Rocky Hill, NJ, USA) in serum-free DMEM for $10 \mathrm{~min}$. Cells were fixed with $2 \%$ Paraformaldehyde (PFA; EMD) in phosphate-buffered saline (PBS) for $20 \mathrm{~min}$ and excess PFA was neutralized with $0.1 \mathrm{M}$ Glycine/PBS for $5 \mathrm{~min}$. Cells were briefly washed with Glycine/PBS and permeabilized with $0.5 \%$ Triton $\mathrm{X}-100 / \mathrm{PBS}$ for $10 \mathrm{~min}$ at room temperature, blocked with 5\% BSA solution in PBS for $30 \mathrm{~min}$ and subsequently incubated overnight at $4{ }^{\circ} \mathrm{C}$ with primary antibodies in blocking solution, at dilutions of 1:50 for both Tid-1 and Met antibodies. Cells were washed extensively with IF Wash Buffer (130 mm NaCl, $7 \mathrm{~mm} \mathrm{Na} \mathrm{HPO}_{4}, 3.5 \mathrm{~mm}$ $\mathrm{NaH}_{2} \mathrm{PO}_{4}, 7.7 \mathrm{~mm} \mathrm{NaN}, 0.1 \%$ BSA, $0.2 \%$ Triton X-100, $0.05 \%$ Tween-20) and incubated with secondary antibodies (AlexaFluor488 (Anti-Rabbit; Invitrogen, Burlington, ON, Canada) and Cy3 (Anti-Mouse; Jackson ImmunoResearch, West Grove, PA, USA) at 1:200) in blocking solution (5\% $\mathrm{BSA} / \mathrm{PBS}$ ) for $1 \mathrm{~h}$ at room temperature. Cells were washed with IF wash Buffer and mounted with DAPI-containing VECTASHIELD Mounting Medium. Images were captured using a Zeiss LSM 410 Laser Scanning Confocal Microscope (Zeiss, Toronto, ON, Canada) and analyzed with the appropriate software.

Transwell migration assay

786-0 RCC cells were resuspended in $500 \mu \mathrm{L}$ of serum-free DMEM supplemented with $0.5 \%$ BSA and plated in the upper chamber of an uncoated transwell chamber with an $8-\mu \mathrm{m}$ pore size (BD Biosciences). The lower chamber contained $500 \mu \mathrm{l}$ of serum-free DMEM supplemented with $0.5 \%$ BSA and $40 \mathrm{ng} / \mathrm{ml} \mathrm{HGF}$ as a chemoattractant. After $24 \mathrm{~h}$ of incubation, the cells that migrated through the filter were stained (Diff-Quik). Filters were mounted on slides with immersion 
oil and photographed using a Nikon TE2000 Eclipse (Nikon, Mississauga, ON, Canada). The number of cells that migrated was counted in five individual fields/filter. Data shown represents pooled data from three independent experiments.

\section{Wound closure assay}

786-0 cells were grown to confluency and serum-starved overnight in serum-free DMEM. A 200- $\mu$ l pipette tip was used to make a uniform wound in the cell layer. Cells were washed thoroughly with serum-free DMEM and fresh serumfree DMEM was added at $0 \mathrm{~h}$ with or without the addition of $5 \mathrm{ng} / \mathrm{ml} \mathrm{HGF}$ where indicated. A Nikon TE2000 Eclipse was used to photograph specific fields of the wound at the indicated time points. These photographs were used to measure the width of the wound using Simple PCI software (Mississauga, $\mathrm{ON}$, Canada) and percentage wound closure was calculated using the following equation: \% wound closure $=(1-$ wound width at time $\times$ /wound width at $0 \mathrm{~h}) \times 100$. Data shown represents pooled data from three independent experiments. An unpaired $t$-test was used to calculate significance of either hTid- $1_{S}$ or hTid- $1_{L}$ overexpressing cells compared with the control at each time point. A $P$ value of $<0.05$ was considered significant.

\section{Cell proliferation assay}

Cells were infected with Ad-GFP, Ad-hTid-1 ${ }_{S}$ or Ad-hTid-1 ${ }_{\mathrm{L}}$. The following day, 2500 cells were seeded/well of 24 -well plates. Cells were serum-starved overnight, and left untreated or stimulated with the indicated concentration of HGF. The media was changed every $48 \mathrm{~h}$ to fresh starvation media

\section{References}

Ahn BY, Trinh DL, Zajchowski LD, Lee B, Elwi AN, Kim SW. (2010). Tid1 is a new regulator of p53 mitochondrial translocation and apoptosis in cancer. Oncogene 29: 1155-1166.

Bae MK, Jeong JW, Kim SH, Kim SY, Kang HJ, Kim DM et al. (2005). Tid-1 interacts with the von Hippel-Lindau protein and modulates angiogenesis by destabilization of HIF-1alpha. Cancer Res 65: 2520-2525.

Banecki B, Liberek K, Wall D, Wawrzynow A, Georgopoulos C, Bertoli E et al. (1996). Structure-function analysis of the zinc finger region of the DnaJ molecular chaperone. J Biol Chem 271: 14840-14848.

Benvenuti S, Comoglio PM. (2007). The MET receptor tyrosine kinase in invasion and metastasis. $J$ Cell Physiol 213: 316-325.

Birchmeier C, Birchmeier W, Gherardi E, Vande Woude GF. (2003). Met, metastasis, motility and more. Nat Rev Mol Cell Biol 4: 915-925.

Chang H-CJ, Nathan DF, Lindquist S. (1997). In vivo analysis of the Hsp90 cochaperone Sti1 (p60). Mol Cell Biol 17: 318-325.

Chen CY, Chiou SH, Huang CY, Jan CI, Lin SC, Hu WY et al. (2009). Tid1 functions as a tumour suppressor in head and neck squamous cell carcinoma. $J$ Pathol 219: 347-355.

Cheng H, Cenciarelli C, Nelkin G, Tsan R, Fan D, Cheng-Mayer C et al. (2005). Molecular mechanism of hTid-1, the human homolog of Drosophila tumor suppressor 1(2)Tid, in the regulation of NF-kappaB activity and suppression of tumor growth. Mol Cell Biol. 25: 44-59.

Cheng H, Cenciarelli C, Tao M, Parks WP, Cheng-Mayer C. (2002). HTLV-1 Tax-associated hTid-1, a human DnaJ protein, is a repressor of Ikappa B kinase beta subunit. J Biol Chem 277: 20605-20610.

Duina AA, Chang H-C, Marsh JA, Lindquist S, Gaber RF. (1996). A cyclophilin function in Hsp90-dependent signal transduction. Science 274: 1713-1715.

Edwards KM, Munger K. (2004). Depletion of physiological levels of the human TID1 protein renders cancer cell lines resistant to apoptosis mediated by multiple exogenous stimuli. Oncogene 23: 8419-8431. with HGF. Cells were counted manually at the indicated time points after infection using trypan blue exclusion. Growth assays were performed at least three independent times.

\section{Densitometric analysis}

Densitometric analysis of western blot results was performed using Scion Image software (Scion Corp., Frederick, MD, USA). The pixel densities for both the phosphorylated protein and total protein as indicated were determined and were expressed as a ratio of phosphorylated protein:total protein.

\section{Statistical analysis}

A student's $t$-test was used to compare each experimental condition with the control condition. A $P$-value of $<0.05$ was considered significant.

\section{Conflict of interest}

The authors declare no conflict of interest.

\section{Acknowledgements}

This work was supported by a grant from the National Cancer Institure of Canada ( to MRA). ER was supported by an Ontario Graduate Scholarship and CML by a Canadian Institutes of Health Research Master's Award. We thank Kevin Conway for assistance with immunofluorescence microscopy.
Ferracini R, Di Renzo MF, Scotlandi K, Baldini N, Olivero M, Lollini $\mathrm{P}$ et al. (1995). The Met/HGF receptor is over-expressed in human osteosarcomas and is activated by either a paracrine or an autocrine circuit. Oncogene 10: 739-749.

Freeman BC, Toft DO, Morimoto RI. (1996). Molecular chaperone machines: chaperone activities of the cyclophilin Cyp-40 and the steroid aporeceptor-associated protein p23. Science 274: 1718-1720.

Frydman J, Nimmesgern E, Ohtsuka K, Hartl FU. (1994). Folding of nascent polypeptide chains in a high molecular mass assembly with molecular chaperones. Nature 370: 111-117.

Hammond DE, Urbe S, Vande Woude GF, Clague MJ. (2001). Downregulation of MET, the receptor for hepatocyte growth factor. Oncogene 20: 2761-2770.

Hartl FU. (1996). Molecular chaperones in cellular protein folding Nature 381: 571-579.

Hov H, Holt RU, Ro TB, Fagerli UM, Hjorth-Hansen H, Baykov V et al. (2004). A selective c-met inhibitor blocks an autocrine hepatocyte growth factor growth loop in ANBL-6 cells and prevents migration and adhesion of myeloma cells. Clin Cancer Res 10: 6686-6694.

Iliopoulos O, Levy AP, Jiang C, Kaelin Jr WG, Goldberg MA. (1996). Negative regulation of hypoxia-inducible genes by the von HippelLindau protein. Proc Natl Acad Sci USA 93: 10595-10599.

Jolly C, Morimoto RI. (2000). Role of the heat shock response and molecular chaperones in oncogenesis and cell death. $J$ Natl Cancer Inst 92: 1564-1572.

Kim SW, Chao TH, Xiang R, Lo JF, Campbell MJ, Fearns C et al. (2004). Tid1, the human homologue of a Drosophila tumor suppressor, reduces the malignant activity of ErbB-2 in carcinoma cells. Cancer Res 64: 7732-7739.

Koga F, Tsutsumi S, Neckers LM. (2007). Low dose geldanamycin inhibits hepatocyte growth factor and hypoxia-stimulated invasion of cancer cells. Cell Cycle 6: 1393-1402. 
Koochekpour S, Jeffers M, Wang PH, Gong C, Taylor GA, Roessler LM et al. (1999). The von Hippel-Lindau tumor suppressor gene inhibits hepatocyte growth factor/scatter factor-induced invasion and branching morphogenesis in renal carcinoma cells. Mol Cell Biol 19: 5902-5912.

Kurzik-Dumke U, Czaja J. (2007). Htid-1, the human homolog of the Drosophila melanogaster 1(2)tid tumor suppressor, defines a novel physiological role of APC. Cell Signal. 19: 1973-1985.

Kurzik-Dumke U, Gundacker D, Renthrop M, Gateff E. (1995). Tumor suppression in Drosophila is causally related to the function of the lethal(2)tumorous imaginal discs gene, a dnaJ homolog. Developmental Genetics 16: 64-76.

Kurzik-Dumke U, Phannavong B, Gundacker D, Gateff E. (1992). Genetic, cytogenic and developmental analysis of the Drosophila melanogaster tumor supressor gene lethal (2) tumorous imaginal discs (1(2)tid). Differentiation 51: 91-104.

Lesko E, Gozdzik J, Kijowski J, Jenner B, Wiecha O, Majka M. (2007). HSP90 antagonist, geldanamycin, inhibits proliferation, induces apoptosis and blocks migration of rhabdomyosarcoma cells in vitro and seeding into bone marrow in vivo. Anticancer Drugs 18: 1173-1181.

Linnoila J, Wang Y, Yao Y, Wang ZZ. (2008). A mammalian homolog of Drosophila tumorous imaginal discs, Tid1, mediates agrin signaling at the neuromuscular junction. Neuron 60: 625-641.

Liu HY, MacDonald JI, Hryciw T, Li C, Meakin SO. (2005). Human tumorous imaginal disc 1 (TID1) associates with Trk receptor tyrosine kinases and regulates neurite outgrowth in nnr5-TrkA cells. J Biol Chem 280: 19461-19471.

Maulik G, Kijima T, Ma PC, Ghosh SK, Lin J, Shapiro GI et al. (2002). Modulation of the c-Met/hepatocyte growth factor pathway in small cell lung cancer. Clin Cancer Res 8: 620-627.

Nakaigawa N, Yao M, Baba M, Kato S, Kishida T, Hattori K et al. (2006). Inactivation of von Hippel-Lindau gene induces constitutive phosphorylation of MET protein in clear cell renal carcinoma. Cancer Res. 66: 3699-3705.

Natali PG, Prat M, Nicotra MR, Bigotti A, Olivero M, Comoglio PM et al. (1996). Overexpression of the met/HGF receptor in renal cell carcinomas. Int J Cancer 69: 212-217.

Ohh M, Takagi Y, Aso T, Stebbins CE, Pavletich NP, Zbar B, Conaway RC et al. (1999). Synthetic peptides define critical contacts between elongin $\mathrm{C}$, elongin $\mathrm{B}$, and the von Hippel-Lindau protein. $J$ Clin Invest 104: 1583-1591.

Pennacchietti S, Michieli P, Galluzzo M, Mazzone M, Giordano S, Comoglio PM. (2003). Hypoxia promotes invasive growth by transcriptional activation of the met protooncogene. Cancer Cell 3: $347-361$

Peruzzi B, Athauda G, Bottaro DP. (2006). The von Hippel-Lindau tumor suppressor gene product represses oncogenic beta-catenin signaling in renal carcinoma cells. Proc Natl Acad Sci USA 103: 14531-14536.

Peruzzi B, Bottaro DP. (2006). Beta-catenin signaling: linking renal cel carcinoma and polycystic kidney disease. Cell Cycle 5: 2839-2841.

Pratt WB, Toft DO. (2003). Regulation of signaling protein function and trafficking by the hsp90/hsp70-based chaperone machinery. Exp Biol Med (Maywood) 228: 111-133.

Qian J, Perchiniak EM, Sun K, Groden J. (2010). The mitochondrial protein hTID-1 partners with the caspase-cleaved adenomatous polyposis cell tumor suppressor to facilitate apoptosis. Gastroenterology 138: 1418-1428.
Rong S, Segal S, Anver M, Resau JH, Vande Woude GF. (1994). Invasiveness and metastasis of NIH 3T3 cells induced by Met-hepatocyte growth factor/scatter factor autocrine stimulation. Proc Natl Acad Sci USA 91: 4731-4735.

Sangwan V, Paliouras GN, Abella JV, Dube N, Monast A, Tremblay ML et al. (2008). Regulation of the Met Receptortyrosine kinase by the protein-tyrosine phosphatase 1B and T-cell phosphatase. J Biol Chem 283: 34374-34383.

Sarkar S, Pollack BP, Lin KT, Kotenko SV, Cook JR, Lewis A et al. (2001). hTid-1, a human DnaJ protein, modulates the interferon signaling pathway. J Biol Chem 276: 49034-49042.

Schilling B, De-Medina T, Syken J, Vidal M, Munger K. (1998). A novel human DnaJ protein, hTid-1, a homolog of the Drosophila tumor suppressor protein Tid56, can interact with the human papillomavirus type 16 E7 oncoprotein. Virology 247: 74-85.

Schaaf CP, Benzing J, Schmitt T, Erz DH, Tewes M, Bartram CR et al. (2005). Novel interaction partners of the TPR/MET tyrosine kinase. FASEB J 19: 267-269.

Syken J, De-Medina T, Munger K. (1999). TID1, a human homolog of the Drosophila tumor suppressor 1(2)tid, encodes two mitochondrial modulators of apoptosis with opposing functions. Proc Natl Acad Sci USA 96: 8499-8504.

Syken J, Macian F, Agarwal S, Rao A, Munger K. (2003). TID1, a mammalian homologue of the drosophila tumor suppressor lethal(2) tumorous imaginal discs, regulates activation-induced cell death in Th2 cells. Oncogene 22: 4636-4641.

Trentin GA, He Y, Wu DC, Tang D, Rozakis-Adcock M. (2004). Identification of a hTid-1 mutation which sensitizes gliomas to apoptosis. FEBS Lett. 578: 323-330.

Trentin GA, Yin X, Tahir S, Lhotak S, Farhang-Fallah J, Li Y et al. (2001). A mouse homologue of the Drosophila tumor suppressor 1(2)tid gene defines a novel Ras GTPase-activating protein (RasGAP)-binding protein. J Biol Chem 276: 13087-13095.

Wakabayashi Y, Mao JH, Brown K, Girardi M, Balmain A. (2007). Promotion of Hras-induced squamous carcinomas by a polymorphic variant of the Patched gene in FVB mice. Nature 445: 761-765.

Wall D, Zylicz M, Georgopoulous C. (1994). The NH2-terminal 108 amino acids of the Escherichia coli DnaJ protein stimulate the ATPase activity of DnaK and are sufficient for lamba replication. J. Biol. Chem. 269: 5446-5451.

Webb CP, Hose CD, Koochekpour S, Jeffers M, Oskarsson M, Sausville $\mathrm{E}$ et al. (2000). The geldanamycins are potent inhibitors of the hepatocyte growth factor/scatter factor-met-urokinase plasminogen activator-plasmin proteolytic network. Cancer Res 60: 342-349.

Yin X, Rozakis-Adcock M. (2001). Genomic organization and expression of the human tumorous imaginal disc (TID1) gene. Gene 278: 201-210.

Zia MK, Rmali KA, Watkins G, Mansel RE, Jiang WG. (2007). The expression of the von Hippel-Lindau gene product and its impact on invasiveness of human breast cancer cells. Int J Mol Med 20: 605-611.

This work is licensed under the Creative Commons Attribution-NonCommercial-No Derivative Works 3.0 Unported License. To view a copy of this license, visit http://creativecommons.org/licenses/by-nc-nd/3.0/ 